\title{
Evolution of Neural Symmetry and its Coupled Alignment to Body Plan Morphology
}

\author{
Ben Jones \\ Natural Computation Group \\ School of Computer Science \\ University of Birmingham, UK \\ ben@bhjones.com
}

\author{
Andrea Soltoggio \\ Research Institute for \\ Cognition and Robotics \\ Bielefeld University, Germany \\ asoltogg@cor-lab.uni- \\ bielefeld.de \\ Xin Yao \\ Natural Computation Group \\ School of Computer Science \\ University of Birmingham, UK \\ X.Yao@cs.bham.ac.uk
}

\author{
Bernhard Sendhoff \\ Honda Research Institute \\ Europe $\mathrm{GmbH}$ \\ Offenbach/Main \\ Germany \\ bs@honda-ri.de
}

\begin{abstract}
Body morphology is thought to have heavily influenced the evolution of neural architecture. However, the extent of this interaction and its underlying principles are largely unclear. To help us elucidate these principles, we examine the artificial evolution of a hypothetical nervous system embedded in a fish-inspired animat. The aim is to observe the evolution of neural structures in relation to both body morphology and required motor primitives. Our investigations reveal that increasing the pressure to evolve a wider range of movements also results in higher levels of neural symmetry. We further examine how different body shapes affect the evolution of neural structure; we find that, in order to achieve optimal movements, the neural structure integrates and compensates for asymmetrical body morphology. Our study clearly indicates that different parts of the animat - specifically, nervous system and body plan - evolve in concert with and become highly functional with respect to the other parts. The autonomous emergence of morphological and neural computation in this model contributes to unveiling the surprisingly strong coupling of such systems in nature.
\end{abstract}

\section{Categories and Subject Descriptors}

I.2.6 [Learning]: Metrics-Connectionism and Neural Nets

\section{General Terms}

Algorithms

\section{Keywords}

Neuroevolution, Body Symmetry, Morphology, Motor Primitives, Artificial Life, Soft Robotics

Permission to make digital or hard copies of all or part of this work for personal or classroom use is granted without fee provided that copies are not made or distributed for profit or commercial advantage and that copies bear this notice and the full citation on the first page. To copy otherwise, to republish, to post on servers or to redistribute to lists, requires prior specific permission and/or a fee.

GECCO'11, July 12-16, 2011, Dublin, Ireland.

Copyright 2011 ACM 978-1-4503-0557-0/11/07 ...\$10.00.

\section{INTRODUCTION}

Genetic evidence reveals that animals having distinctive types of body symmetry are in many ways related [17, 18]. An abundance of common genetic markers (certain homeobox genes [24, 8]) present in both the Hydra, a very primitive radially symmetric jellyfish, and the more advanced Bilateria have been identified as being associated with pattern formation. It is conjectured that diversity arose from a common ancestor $[15,10,16]$ with a nervous system that evolved in concert with the body plan [9].

Over the years, several bodies of research have attempted to elucidate the mechanisms of nervous system evolution in relation to the neurological basis of behaviour. Ghysen [10] asks how functional coherence can arise in many different lifeforms even though their respective lifestyles and habitats are richly diverse. Holland [11] explores the genetic basis of functional components within the central nervous system by searching for common genetic homologues. Murakami et al. [19] considers the lamprey while exploring how the developmental plan has evolved and arisen in more advanced vertebrates.

Seemingly complex behaviour can also originate from relatively simple neural structures. As first shown in 'Vehicles: Experiments in Synthetic Psychology', Braitenberg [7] posited several thoughtexperiments in which the mechanistic and behavioural properties of a simple agent were progressively complexified. Whilst these mechanisms were grounded in simplistic sensory-motor pathways, they were nevertheless able to result in a complex behavioural repertoire. In such a spirit of simplicity, yet with the aim of biological relevancy, models have become progressively centric to biophysically realistic behaviour. The work of Karl Sims [25], who explored how abstract neural control systems could implement a rich set of animal-like behaviours in several artificial creatures, is a good example. Others (e.g. [5, 4]) have further asked how brain and body can evolve in a coupled fashion. Together with neural control, the body and environment are also argued to be core to the evolutionary process in so-called "embodied intelligence", see e.g. [28].

The model of Schramm et al. [22] addresses body morphology and control as a coupled process. Their model employs an evolved genetic regulatory network (GRN) that controls cell growth. Cells, which in the model are physical point masses, are grown and connected together with springs. Actuation of lateral springs then provides movement. A variety of body morphologies, coupled to the 
control mechanism, are then demonstrated to yield different types of movement. The work of Jones et al. [13] studies how motor neuron configurations evolve to become bilaterally symmetric. In an elongated animat, motors are distributed around the length of the body and can evolve into both radial and bilateral configurations. In the swimming task of that study, evolution led motor pairs to assume bilateral configurations. The efficiency of bilateral morphology in directional movement is further stressed in [6]. More recently, Bongard [3] examined the evolution of body plan morphogenesis finding that when lifetime developmental changes are permitted, greater robustness pervades behaviour generation.

In this paper, we address how neural structure evolves to control an anguilliform agent required to perform a set of different motor skills. Under such enhanced and realistic scenarios, symmetrical properties and the integration with body morphology have been subject to analysis. During evolution, neural structure changes due to the genetically determined positions of connections and neurons. Connection weights are computed as a function of interneuronal Euclidean distance. The large search space of all possible neural structures has enabled us to observe the autonomous and unbiased evolution of the coupled control system.

A major point in this study is how the need to evolve different motor skills affects the structural distribution and symmetry of the neural control system. In a first scenario the agent is required to undertake fast, efficient forward motion: this task elicits the evolution of only one oscillatory-type motor primitive. In a second scenario, the agent is required to perform left and right turns while maintaining forward motion. This task is more complex and requires a correct synergy between motor primitives. In a third scenario, we then impose asymmetry onto the body plan and again investigate how this affects the evolution of neural structure. The main finding is that an increased number of motor primitives promotes the evolution of highly symmetrical neural structures. We hypothesize that higher movement requirements lead evolution to fine-tuning the underlying movement mechanism. However, when the body morphology is further asymmetrically structured, the neural architecture adapts and compensates to achieve optimality in the required set of movements.

The rest of this paper is laid out as follows. In Section 2, the model and encompassing simulation environment are described. Section 3 then outlines the experimental setup. Results are presented in Section 4. We conclude in Section 5.

\section{MODEL}

A fish-like animat with a neural controller inspired by primitive neural organisation has been implemented. The animat's body morphology is three dimensional and is composed of springs as shown in Fig. 1(upper). The width of the animat is 1.26 and the length of a body segment is 3.525 ; the animat is composed of 15 body segments meaning that the length of the whole animat is 52.875 . These values are not those of a particular animal, nor do they represent particular units, nevertheless, they approximately reproduce the proportions of an elongated swimming animal. An abstract model of water force as described in $[12,23]$ is further modelled to allow simulated movement through a liquid environment.

\subsection{Motor system}

Fig. 1 shows how bilaterally arranged pairs of motor neurons actuate triplets of body segments. This arrangement allows a single motor to induce a degree of curvature in the body, see Fig. 1(lower). The level of compression applied during this actuation process is proportional to the output activation of the respective motor neuron.

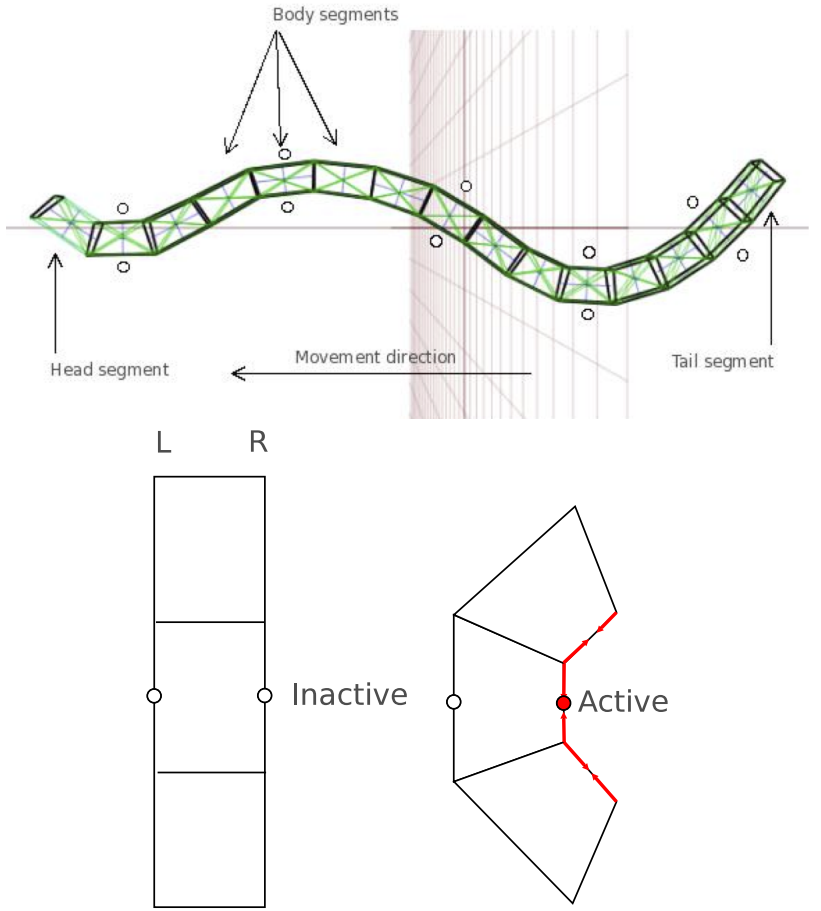

Figure 1: Upper: Visualisation of the model agent used in the study. The lateral circles depict the positions of motor neurons which serve to actuate sets of lateral springs. Lower: A diagram showing how motor pairs actuate a triplet of body segments. A pair of motors per triplet allows for a natural generation of body curvature when one of those motors becomes active.

When particular springs are compressed, geometry is altered and movement is generated.

\subsection{Computational neural system}

The neural dynamics are modelled with leaky integrators. The membrane potential $u_{j}$ of a neuron is [2]:

$$
\frac{d u_{j}}{d t}=\frac{1}{\tau_{j}}\left(-u_{j}+\sum_{i=1}^{C} w_{j i} a_{i}+I_{j}\right)
$$

where $\tau_{j}$ is the neuron discharge time constant, $w$ is a vector of presynaptic connection weights and $I_{j}$ is an external input current. The activity $a_{i}$ of a neuron is computed as $\tanh \left(u_{i}\right)$. If a neuron is inhibitory then all of its outgoing connection weights are negative. Motor neurons are excitatory and have a time constant value of 1. This is to model biological muscles that have only periods of contraction (excitation) or relaxation. The output of a given motor $m$ is computed as $1 / 1+\exp \left(-u_{m}-1\right)$. This yields a positive value which is then used in driving the compression levels of the associated springs.

\subsection{Architectural neural system}

The neural system consists of 22 neurons: 10 motor neurons, i.e. two for each triplet of body segments, an equal number of descending neurons and 2 head-CPG neurons as shown in Fig. 2. This setting was found to be sufficient for generating the required set of movements whilst maintaining at the same time a minimalistic approach. Connectivity is established according to boolean genes except for those connections within the head-CPG structure which 


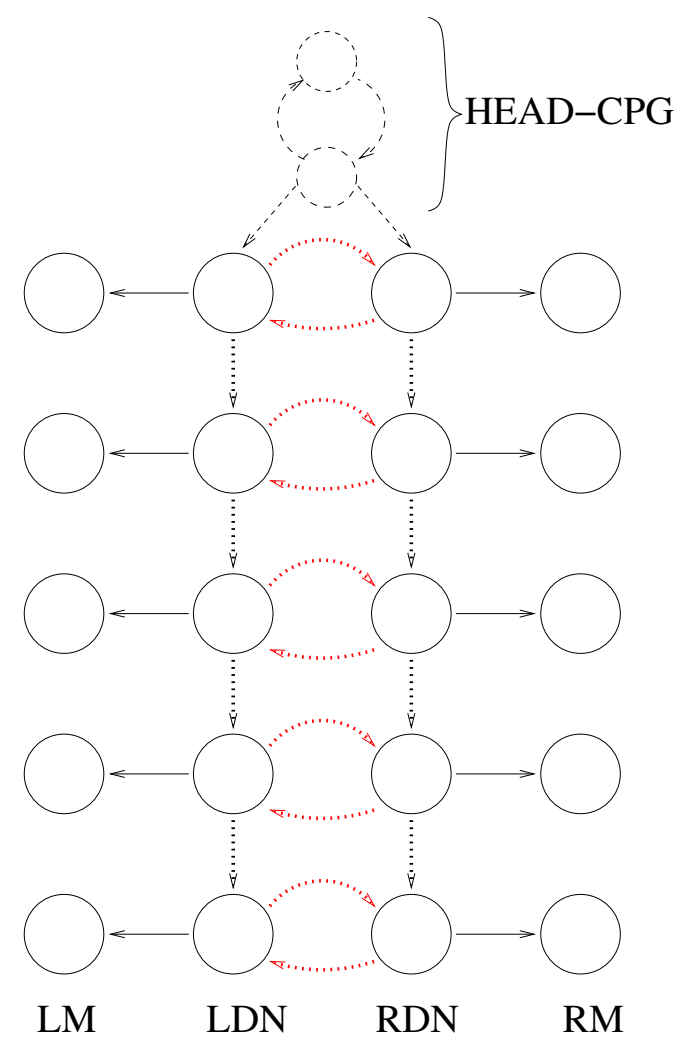

Figure 2: A schematic of the agent's neural control system. LM, LDN, RDN and RM refer to Left Motors, Left Descending Neurons, Right Descending Neurons and Right Motors respectively. Dotted lines signify evolved connections. The HEADCPG is further considered a single functional module - including both neurons and connections; its presence in the phenotype is determined by a single boolean gene.

is considered a whole functional module. Connections between the descending neurons are determined as follows: a connection from a left descending neuron $\left(\mathrm{LDN}_{i}\right)$ to the equivalent right descending neuron $\left(\mathrm{RDN}_{i}\right)$ is mirrored with a connection from $\mathrm{RDN}_{i}$ to $\mathrm{LDN}_{i}$. This permits the formation of coupled oscillators. The presence of individual descending connections is determined by a set of boolean genes.

\subsubsection{Synaptic connectivity}

An important aspect of this model is the effect that neural structure has on synaptic efficacy. Specifically, weight connections are derived from the interneuronal Euclidean distance. The closer two neurons are together, the higher the weight value between them,

$$
w_{i j}=\xi / d_{i j}
$$

where $\xi$ has been empirically set to 20.0 to ensure that a suitable range of weights can be generated. Accordingly, the position of a neuron is changed through a process of simulated evolution. In other words, the weight values are implicitly represented. Only the positions of the descending and head-CPG neurons are evolved. The positions of the motor neurons remain fixed. The distance of a given descending neuron from the centre of the animat (i.e. in the horizontal x-plane) and the positions of the head-CPG neurons along the vertical y-dimension are determined by evolution.
This mechanism for the head triplet of body segments is depicted in Fig. 3.

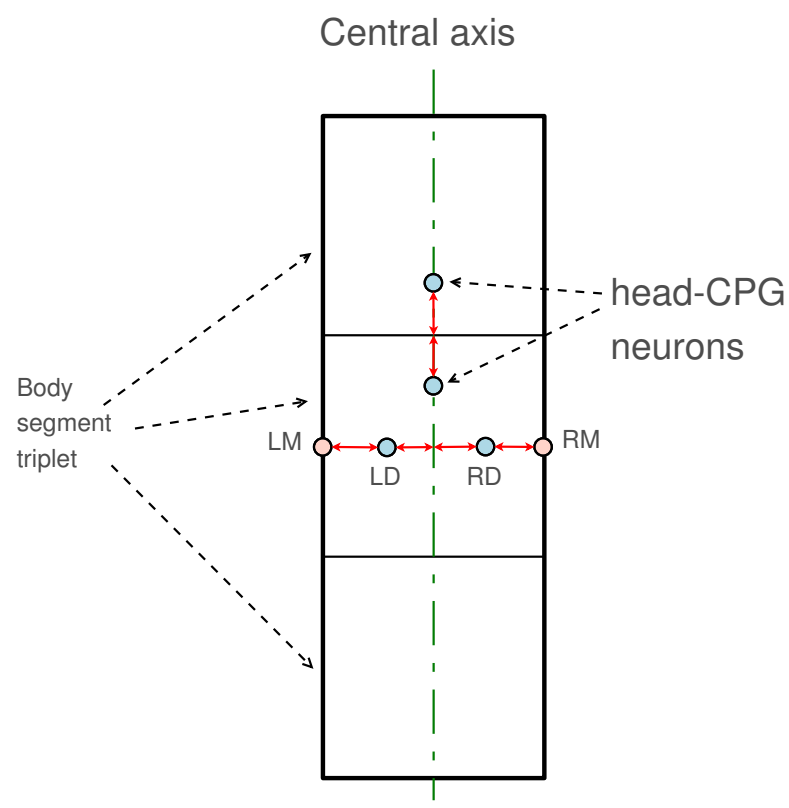

Figure 3: The manner in which neuron positions are evolved as shown for the first triplet of body segments. The x-dimensions of the descending neurons (LD and RD) can move closer or further from their respective motor neurons (LM and RM). The head-CPG neurons, which are only present within the head triplet, move towards or away from each other along the ydimension. Interneuronal distances are used in deriving weight connection values.

\subsubsection{Turning mechanism}

The agent is endowed with a pair of sensors located at the top of the head segment. The sensor that is found to be closest to an environmental target is designated the winner and becomes active whilst the other is designated the loser and remains dormant. In order for the animat to turn, one of its sides needs to be actuated more strongly. To do this, the winning sensor affects a ratio of left and right force magnitudes LF:RF that are applied to the left and right actuated springs respectively. When the ratio is biased towards either LF or RF, as determined by the winning sensor, the body geometry is altered. The ratio is initialised to $1: 1$ meaning that initially there is no bias towards the left or right. Throughout a simulation, this ratio changes according to the above described winner-takes-all strategy, as shown:

$$
\mathrm{f}\left(\delta_{\mathrm{l}}, \delta_{\mathrm{r}}\right)= \begin{cases}\delta_{\mathrm{r}}=0.01 \cdot n_{r} ; \delta_{\mathrm{l}}=-0.01 \cdot n_{l} & \mathrm{RS} \\ \delta_{\mathrm{r}}=-0.01 \cdot n_{r} ; \delta_{\mathrm{l}}=0.01 \cdot n_{l} & \text { LS }\end{cases}
$$

where $\delta_{l}$ and $\delta_{r}$ are the updates to LF and RF respectively and $n_{l}$ and $n_{r}$ are the outputs of the left and right motor neurons respectively. Thus the force magnitude is increased for the winning side of the animat and decreased for the losing side. This ratio changes smoothly due to the effect of the factor 0.01 in Eq. 3. The agent can turn smoothly without hindrance to the stability of the physics model; during testing, it was found that too sharp a turn could cause the animat to fold in on itself. A visualisation of the turning mechanism is presented later in the results section. 


\section{EXPERIMENTAL SETUP}

The motivation of these experiments is to study the effect that body morphology has on the evolution of neural structure and moreover, how a requirement for different motor primitives can affect such structure. In order to do this, the following three sets of experiments were performed:

(a) the agent is required to undertake fast, efficient forward locomotion.

(b) the agent is additionally required to undertake turning behaviour.

(c) as in (a) but with an asymmetric body plan as in Fig. 4.

The first two scenarios, (a) and (b), examine how neural structure evolves in relation to a symmetric body morphology. A natural extension of this is then to ask how an asymmetrical body morphology affects neural structure, i.e. Scenario (c).

The animat is evaluated in a two dimensional plane such that only movements generated by the left and right halves of the agent, as partitioned by the sagittal plane, are evaluated. In Scenarios (a) and (c) the fitness of an agent is determined by how far it can swim in a forwards direction. For Scenario (b), the animat is evaluated three times. For each evaluation, the agent is required to swim towards a target in one of three different locations $(0 \pi, 3 / 2 \pi, \pi / 2$ or north, west and east w.r.t. the starting orientation of the animat) and at a distance that is sufficiently far enough that the animat can never reach it. The sum of these distances is then taken as the fitness measure.

In order to observe the evolution of symmetrical patterns, a measure of neural symmetry was introduced:

$$
\operatorname{sym}=\frac{1}{5} \sum_{i=1}^{5}\left|d\left(\operatorname{LDN}_{i}, c_{i}\right)-d\left(\operatorname{RDN}_{i}, c_{i}\right)\right|
$$

where the function $d_{i}$ is a measure of Euclidean distance. $\mathrm{LDN}_{i}$ and $\mathrm{RDN}_{i}$ represent the positions of the left and right descending neurons respectively; $c_{i}$ represents the position of the centre point along the sagittal plane. An advantage of using this measure is that we are able to observe the process by which symmetry within the neural structure changes during evolution. High levels of neural symmetry will thus have a minimal sym value.
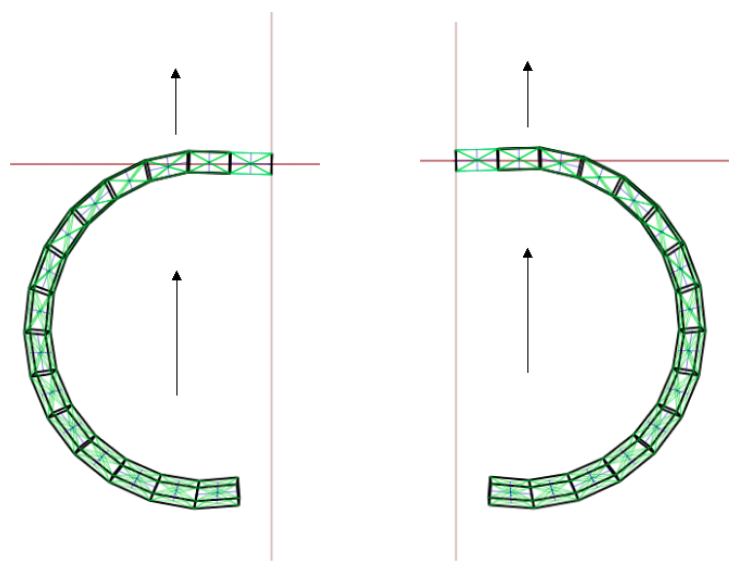

Figure 4: Asymmetric body morphologies: in Scenario (c), the body plan is constrained to adopt one of two body curvatures (curved right or curved left).

\subsection{Evolutionary process}

Artificial evolution is used to evolve a population of 40 individuals. For each scenario, we conducted 50 independently seeded runs except for Scenario (c) in which the number was doubled to include both a left-biased and a right-biased body asymmetry. The genotype and the constraints of the search space are shown in Fig. 5. Note that the value ranges given for NRAD and HY are actually bounded by the geometrical constraints of the animat (refer to Section 2). A maximum of 2200 generations is permitted for each evolutionary run. This number of generations was found during preliminary runs to be sufficient for the fitness to reach a plateau.

\begin{tabular}{|c|c|c|c|c|c|c|}
\hline NI & NTC & head- & DCOP & DES & NRAD & HY \\
$10 / 12$ & $10 / 12$ & 1 & 5 & 8 & 10 & $0 / 2$ \\
BOOL & {$[10,50]$} & BOOL & BOOL & BOOL & {$[0,0.63]$} & {$[0,10.58]$} \\
\hline
\end{tabular}

Figure 5: The agent's genotype. Each box holds the following information: gene type (top); gene count (middle); initialisation range or BOOL if boolean valued (lower). The gene types are: NI (neuron is inhibitory); NTC (neuronal time constants); head-CPG (presence of head-CPG structure); DCOP (coupled connections between descending pairs of neurons); DES (presence of descending connections); NRAD (radius position of descending neuron, that is, the distance of the neuron from the central midline of the animat); HY (y-coordinate position of neuron in head-CPG, if head-CPG structure is present). For NI and NTC, it is possible for there to be 10 or 12 genes; 12 if a head-CPG structure exists. For the same reason, it is further possible for there to be 0 or $2 \mathrm{HY}$ genes.

\subsubsection{Selection mechanism}

The evolutionary process uses a local selection mechanism as described in [21]. The population is placed into a circular array. A tournament scheme is applied to contiguous sub-population neighbourhoods of size 4 . For example, suppose we have a population of size 8 with members [a,b,c,d,e,f,g,h] and the fittest member in the first subset of 4 members $([a, b, c, d])$ is found to be individual $c$ and the fittest member in the second subset of 4 members $([\mathrm{e}, \mathrm{f}, \mathrm{g}, \mathrm{h}])$ is found to be individual $\mathrm{h}$, then the resulting selectees become [c,c,c,c,h,h,h,h]. An offset of size 4 is further used to determine where at the beginning of the array the selection process begins. This ensures a spread in the gene pool, i.e. it enhances diversity.

\subsubsection{Mutation and crossover}

The selectees are then subject to mutation and cross-over operators. The resulting genomes become the offspring members. These offspring members then replace the population members for the subsequent evolutionary generation. In order to create the offspring pool of chromosomes, two population members are chosen at random from the selectee chromosome pool. The evolutionary operators are then applied to these two members and the process repeats until the size of the offspring vector matches the population size. For a given selected pair of chromosomes, genes are probabilistically selected at a rate of 0.01 to be mutated. If they are realvalued, the Gaussian operator $g(0, \sigma)$ is used; each $\sigma$ parameter is self-adapted in the manner described in [1]. If it is boolean-valued, it is simply flipped. Genes are also probabilistically exchanged at a rate of 0.1 . 


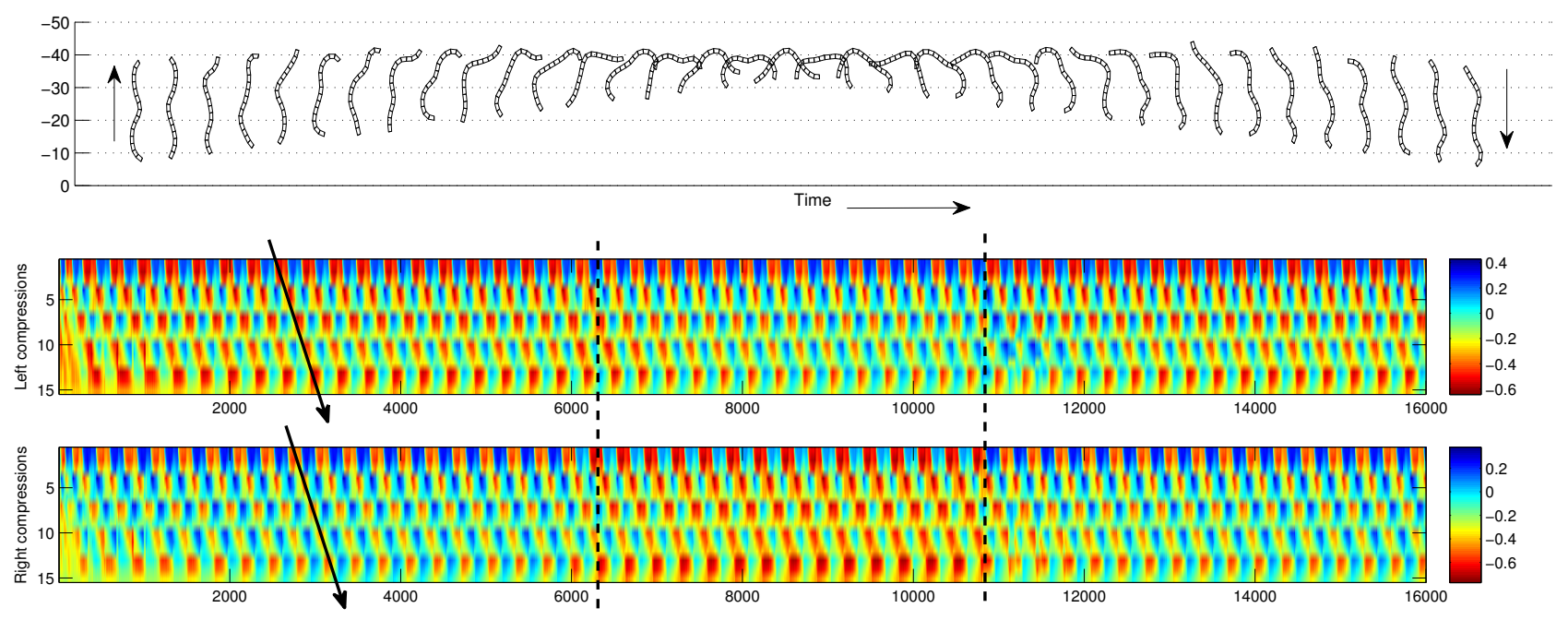

Figure 6: A visualisation of an evolved undulatory agent undertaking a $180^{\circ}$ turn together with a raster plot indicating greater levels of spring compression on the side ipsilateral to turning. Greater levels of compression are marked by deeper levels of 'red' or more negative value. Left-right spring compressions are further shown to occur in 'descending waves', as highlighted by the two diagonal arrows. The two waves are out of phase and correspond to the underlying undulatory movement process.

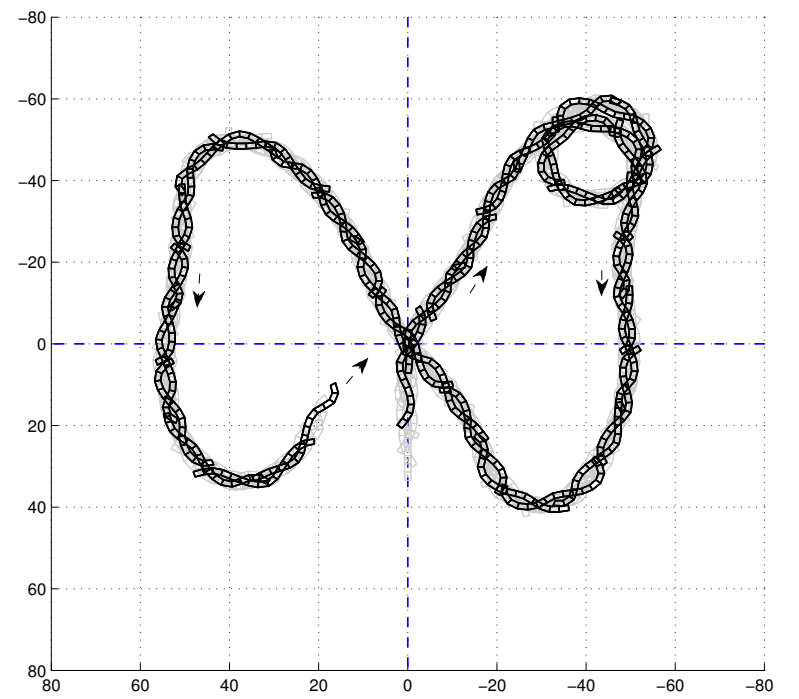

Figure 7: A visualisation showing an evolved agent tracking a target moving in a figure-of-8 pattern. The upper-right circular loop highlights how the agent which has no mechanism to slow down overtook and circled around the target.

\section{RESULTS}

Results from Scenarios (a) and (b) in which the animat had a symmetric body morphology are presented in Section 4.1. Results for Scenario (c) in which the agent had an asymmetrical body morphology are presented in Section 4.2.

\subsection{Scenarios (a) and (b)}

At the end of evolution in Scenario (a), the best performing individuals could undertake efficient forward motion by swimming an average of 3.494 body lengths in 1600 simulation steps as opposed to an average of 0.5898 body lengths before any evolution com-

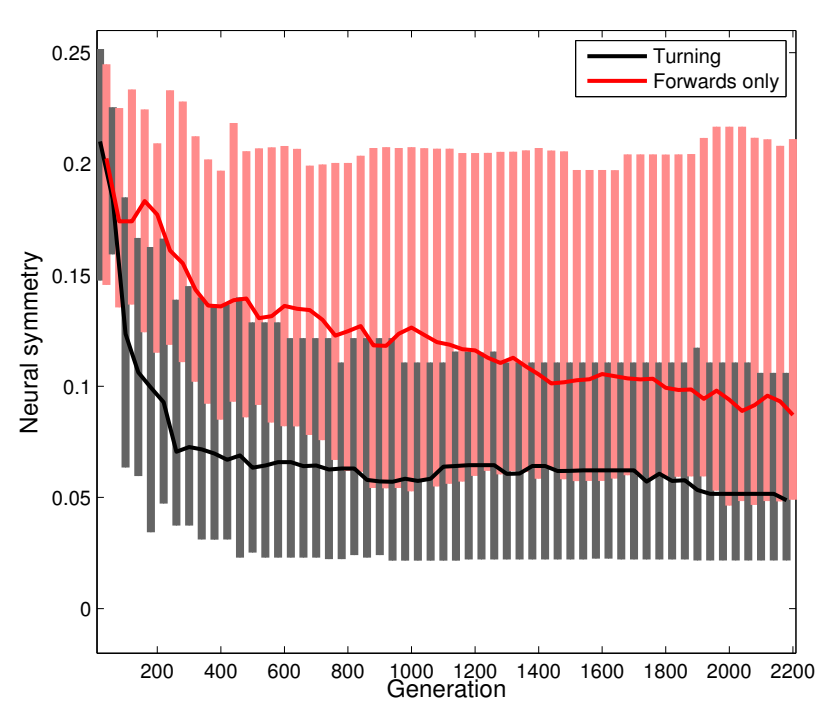

Figure 8: Medians together with upper and lower quartiles of neural symmetry throughout evolution (see Eq. 4 for definition). Symmetry evolves to greater levels (smaller values) in Scenario (b) (forward motion plus turning behaviour) than in Scenario (a) (forward motion only).

menced. This means that the animats which initially moved only marginally evolved the capability of propelling themselves significantly forward.

In Scenario (b), the best performing individuals were further able to turn successfully. An example of this is illustrated in Fig. 6 where an evolved animat is visualised performing a complete uturn. The turning skills of the animat are further demonstrated in Fig. 7 where it is shown tracking a moving target.

The metric defined in Eq. 4 was used to compare neural symme- 

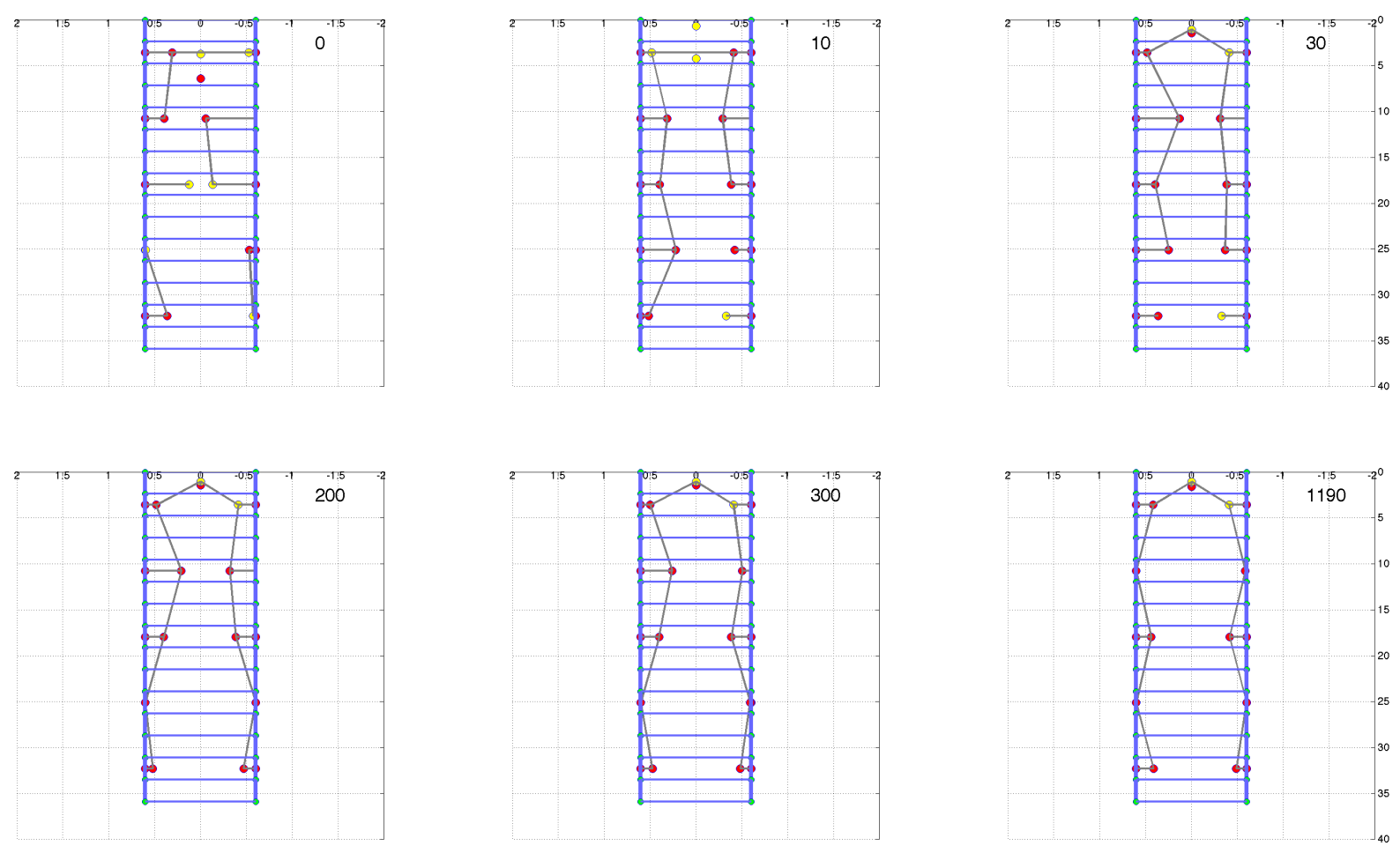

Figure 9: Example of an evolving neural structure: a visualisation showing how the spatial distribution of neurons evolved to become bilaterally symmetric for the fittest individual of a given Scenario (a) experiment. The blue 'ladder-like' structure represents the segmented body morphology. In each visual, the top-right number indicates the current evolutionary generation. Red spheres represent excitatory neurons, yellow spheres inhibitory and grey lines are interneuronal connections. As shown, evolution has resulted in zero coupled connections between the descending neurons, a head-CPG structure and left-right descending pathways that extend through the whole length of the animat.
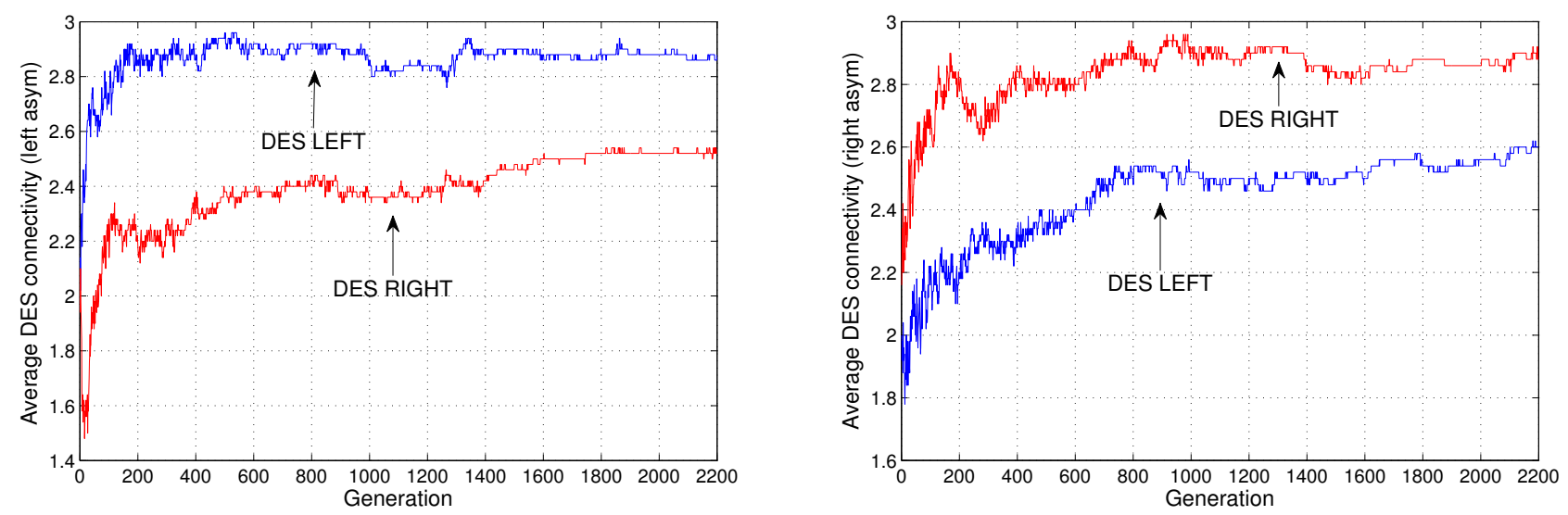

Figure 10: Plots showing the average left/right descending connectivity emergence for left and right biased body asymmetries. As shown, in each case fewer descending connections evolve on the convex side of the body asymmetry. Statistically, differences in left/right DES connectivity in the left plot have a $p$-value of 0.04 , in the right plot 0.07 .

try between both scenarios. The analysis reveals that when the animat is required to turn as well as locomote forwards, neural structures evolve to be more symmetrical, as shown in Fig. 8. In both scenarios, both full descending neural connectivity and a head-CPG structure were observed to evolve and become part of the phenotype. Moreover, Fig. 9 shows that the best individuals evolve to have no coupled connections between descending neurons. This suggests that the required neural dynamics evolved purely from the head-CPG structure. This generates driving oscillatory current through the left and right descending pathways, resulting in a wave of activation propagating through the animat's motor system. This type of architecture in which control becomes regulated 
by a higher-level structure (i.e. the head-CPG unit) and distributed by lower-level components (i.e. descending neuronal pathways) is also shown to be advantageous in a mobile robot platform [20]. An example of an evolving architecture from Scenario (a) is visualised in Fig. 9.

\subsection{Scenario (c)}

With the introduction of a resting body asymmetry, neural architecture evolves in a way that can still endow the agent with functional bilateral control. Statistical analysis indicates that the evolved architectures are biased to compensate the asymmetry of the body plan. Neurons arrange themselves on the concave side of this asymmetry. Moreover, descending connectivity evolves such that there are fewer descending connections on the convex side. These differences in descending connectivity are significant at the p-value levels of 0.04 and 0.07 for the left and right biased body asymmetries respectively as shown in Fig. 10. Two evolved architectures shown in Fig. 11 indicate that this difference in descending connectivity stems from a breakage in the pathway from the headCPG unit on the convex side. This means that propagating oscillations from the head-CPG on this side are prevented from reaching subsequent motors. Further analysis reveals that these motors (enclosed with dashed rectangles in Fig. 11) enter a tonically active state which has the effect of continuously straightening the animat into an upright orientation. On the concave side of the body, the pathway from the head-CPG is intact and activation propagates downwards. The combined effect of propagating oscillations in the concave half and tonic activation in the convex half ultimately enables the animat to maintain a level of forwards locomotive functioning as visualised in Fig. 12.
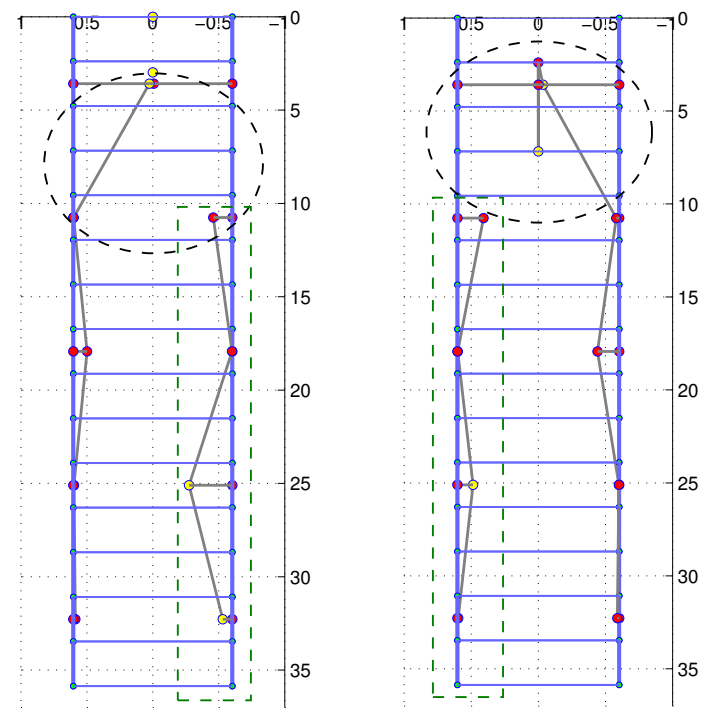

Figure 11: Examples of asymmetrical neural structures for left- and right- biased body asymmetries. The ellipses highlight how descending connectivity from the head-CPG structure is broken on the convex side of the body asymmetry. This largely reflects differences in descending connectivity as plotted in Fig. 10. This results in a lack of oscillatory input current from the head-CPG structure meaning that motor neurons (dashed rectangular regions) yield tonic output. Asymmetrical body morphologies are shown in a straight posture for ease of visualisation.
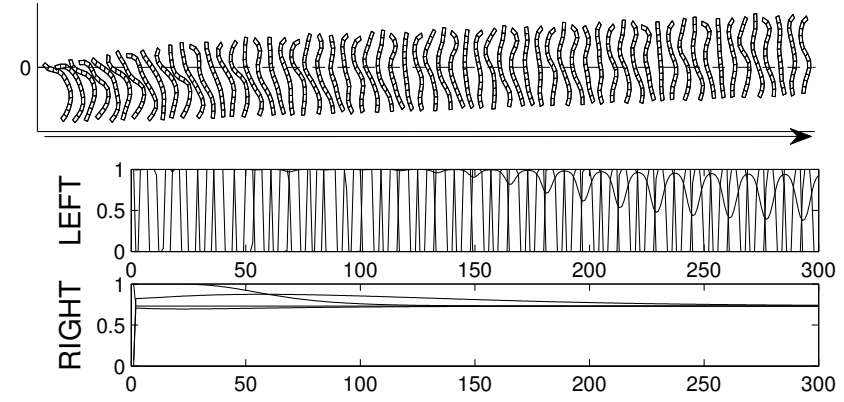

Figure 12: Upper: movement of body: a visualisation showing how for an agent with a left-biased body asymmetry, movement proceeds by initially straightening the animat before it undertakes movement in a forwards direction; arrow indicates time. Lower: the corresponding left/right motor outputs. As shown, the right motors become tonically activated while the left motors oscillate.

\section{CONCLUSIONS}

Our experimental results reveal that neural geometry becomes aligned to body morphology in order to facilitate the generation of motor primitives. The results indicate the following.

1. Artificial evolution favours a symmetrical layout in the neural structure, which appears to improve swimming efficiency.

2. The following simulations in which turning behaviour was additionally required interestingly indicate that, as the motor task becomes richer, an even higher level of symmetry becomes advantageous. This is possibly due to the need for better exploitation of muscle synergies during the turning process.

3. The third experiment, in which the body plan was asymmetrical, demonstrated how the nervous system places itself to compensate for the asymmetrical body. By doing so, forward swimming efficiency is maximised.

Thus the neural structure evolves to exploit both the regularities of the body and the functionality required to achieve locomotion. When the body morphology is symmetrical, the neural geometry exploits the morphological fitness that this can provide in a swimming task; when the body plan is asymmetrical, the neural geometry compensates to achieve the required movements. Our results suggest that, at the evolutionary level, the interplay between neural architecture and body morphology is a fundamental driving mechanism, even at the basic level of these simulations.

In summary, this study addressed the importance of coupling between neural structure and body plan morphology. We have shown this in the context of an artificially evolved fish-inspired animat. Importantly, neural geometry, which is traditionally ignored in the Neuroevolutionary literature, has been shown to be of key importance in terms of how it evolves to relate functionally to body morphology. A model in which the neural structure is embedded within the substrate or body has allowed us to examine the coupling between both. We can sensibly postulate that in animal organisation, the combined evolution of both allows for the exploitation of aspects of morphology and aspects of control; we infer that this strong coupling allows evolution to hone in on a richness of behaviour generation.

Extensions of the model include movements in the coronal plane which can further be evolved in addition to movements in the sagit- 
tal plane. The introduction of energy metrics has also the potential to reveal more efficient neural structures as in [14]. A more advanced neural structural representation might also be adopted, for example, HyperNEAT [27] which - like the model presented in this paper - uses structural information to encode neural connectivity. An advantage of using HyperNEAT is its capacity to yield several plausible emergent properties including symmetry and repetition. At a behavioural level, the simulations can be made to elicit a richer variety of behaviours like variation in speed or simple cognitive tasks. Finally, the modelling of plastic neural connectivity, as those proposed in [26], could reveal more subtle interactions between plastic neural architectures and body morphologies.

\section{Acknowledgements}

The first author would like to thank the Honda Research Institute Europe $\mathrm{GmbH}$ for a grant that supports this research. The research leading to these results has also received funding from the European Community's Seventh Framework Programme FP7/20072013 - Challenge 2 - Cognitive Systems, Interaction, Robotics under grant agreement No 248311 - AMARSi.

\section{REFERENCES}

[1] T. Bäck and H.-P. Schwefel. An overview of evolutionary algorithms for parameter optimization. Evolutionary Computation, 1(1):1-23, 1993.

[2] J. Blynel and D. Floreano. Levels of dynamics and adaptive behavior in evolutionary neural controllers. In From animals to animats: The seventh international conference on simulation of adaptive behavior, pages 272-281, Cambridge, MA, 2002. MIT Press.

[3] J. Bongard. Morphological change in machines accelerates the evolution of robust behavior. Proceedings of the National Academy of Sciences, 108(4):1234-1239, 2011.

[4] J. Bongard and R. Pfeifer. Evolving complete agents using artificial ontogeny. In Morpho-functional Machines: The New Species (Designing Embodied Intelligence), pages 237-258. Springer, Berlin, 2003.

[5] J.C. Bongard. Incremental Approaches to the Combined Evolution of a Robot's Body and Brain. $\mathrm{PhD}$ thesis, Mathematisch-naturwissenschaftlichen Fakultät der Universität Zürich, Zürich, 2003.

[6] J.C. Bongard and C. Paul. Investigating morphological symmetry and locomotive efficiency using virtual embodied evolution. In From Animals to Animats: The Sixth International Conference on Simulation of Adaptive Behavior, Cambridge, MA, 2000. MIT Press.

[7] V. Braitenberg. Vehicles: Experiments in synthetic psychology. MIT Press, Cambridge, Mass, 1984.

[8] M. Broun, S. Sokol, and H.R. Bode. Cngsc, a homologue of goosecoid, participates in the patterning of the head, and is expressed in the organizer region of Hydra. Development, 126:5245-5254, 1999.

[9] J.R. Finnerty. The origins of axial patterning in the metazoa: how old is bilateral symmetry? International Journal of Developmental Biology, 47(7-8):523-529, 2003.

[10] A. Ghysen. The origin and evolution of the nervous system. International Journal of Developmental Biology, 47(8):555-562, 2003.

[11] N.D. Holland. Early central nervous systems evolution: an era of skin brains? Nature reviews, 4(8):617-627, 2003.

[12] B. Jones. The Evolutionary Emergence of Neural Organisation in Computational Models of Primitive
Organisms. $\mathrm{PhD}$ thesis, Computer Science, University of Bimringham, Birmingham, UK, 2010.

[13] B. Jones, Y. Jin, B. Sendhoff, and X. Yao. Evolving functional symmetry in a three dimensional model of an elongated organism. In Artificial Life XI: Proceedings of the Eleventh International Conference on the Simulation and Synthesis of Living Systems, pages 305-312, Cambridge, MA, 2008. MIT Press.

[14] B. Jones, Y. Jin, X. Yao, and B. Sendhoff. Evolution of neural organization in a hydra-like animat. In Proceedings of the 15th International Conference on Neural Information Processing of the Asia-Pacific Neural Network Assembly, volume 1, pages 216-223, Berlin, 2008. Springer.

[15] M. Manuel. Early evolution of symmetry and polarity in metazoan body plans. Comptes Rendus Biologies, 332(2-3):184-209, 2009.

[16] M.Q. Martindale, J.R. Finnerty, and J.Q. Henry. The Radiata and the evolutionary origins of the bilaterian body plan. Molecular Phylogenetics and Evolution, 24(3):358-365, 2002.

[17] H. Meinhardt. The radial-symmetric hydra and the evolution of the bilateral body plan: an old body become a young brain. BioEssays, 24:181-191, 2002.

[18] H. Meinhardt. Different strategies for midline formation in bilaterians. Nature, 5:502-510, 2004.

[19] Y. Murakami, K. Uchida, F.M. Rijli, and S. Kuratani. Evolution of the brain developmental plan: insights from agnathans. Developmental Biology, 280(2):249-259, 2005.

[20] R.W. Paine and J. Tani. How hierarchical control self-organizes in artificial adaptive systems. Adaptive Behavior, 13(3):211-225, 2005.

[21] J. Sarma and K. De Jong. An analysis of the effects of neighborhood size and shape on local selection algorithms. In Parallel Problem Solving from Nature - PPSN IV, volume 1141, pages 236-244, Berlin, 1996. Springer.

[22] L. Schramm, Y. Jin, and B. Sendhoff. Emerged coupling of motor control and morphological development in evolution of multi-cellular animats. In Proceedings of the 10th European Conference on Artificial Life, pages 25-32, Berlin, 2009. Springer.

[23] M. Sfakiotakis and D.P. Tsakiris. Simuun: A simulation environment for undulatory locomotion. International Journal of Modelling and Simulation, 26(4):350-358, 2006.

[24] M.A. Shenk, H.R. Bode, and R.E. Steele. Expression of Cnox-2, a HOM/HOX homeobox gene in hydra, is correlated with axial pattern formation. Development, 117:657-667, 1993.

[25] K. Sims. Evolving 3D morphology and behavior by competition. Artificial Life, 1(4):353-372, 1994.

[26] A. Soltoggio, J.A. Bullinaria, C. Mattiussi, P. Dürr, and D. Floreano. Evolutionary Advantages of Neuromodulated Plasticity in Dynamic, Reward-based Scenarios. In Artificial Life XI: Proceedings of the Eleventh International Conference on the Simulation and Synthesis of Living Systems, pages 569-576, Cambridge, MA, 2008. MIT Press.

[27] K.O. Stanley, D.B. D’Ambrosio, and J. Gauci. A hypercube-based encoding for evolving large-scale neural networks. Artificial Life, 15(2):185-212, 2009.

[28] L. Steels and R. Brooks, editors. The Artificial Life Route to Artificial Intelligence: Building Embodied, Situated Agents. Lawrence Erlbaum Associates, Inc., New Jersey, 1995. 